\title{
Improvement of Localization Algorithm for Wireless Sensor Networks Based on DV-Hop
}

\author{
https://doi.org/10.3991/ijoe.v15i06.9681 \\ Xin Qiao \\ Chaohu University, Anhui, China \\ Fei Chang $(\llbracket)$ \\ Inspur Group Company Limited, Shandong, China \\ fei612@sina.com \\ Jing Ling \\ Chaohu University, Anhui, China
}

\begin{abstract}
DV-Hop localization algorithm is a typical representative of range-free algorithms, which can be applied to large-scale wireless sensor network location monitoring with random distribution of nodes, such as geological hazard monitoring, water pollution monitoring, forest fire monitoring and more. In order to get a higher positioning accuracy, a new method is used to determine the average hop distance of anchor nodes firstly. Secondly, the estimated distances between nodes are calculated by using the correction value corresponding to the average hop distance; finally, in the positioning stage, when the number of anchor nodes is small, the Min-Max (Minimum-Maximum) method is used to obtain the estimated coordinates; on the contrary, the ML (Maximum Likelihood) method is used to calculate the estimated coordinates. One is to reduce the amount of calculation; the other is to keep the accuracy stable. Finally, the Quasi-Newton method is used to iteratively optimize the coordinates of unknown nodes. The results indicate that the accuracy of algorithm is about $15 \%$ $20 \%$ higher than that of the original DV-Hop algorithm.
\end{abstract}

Keywords-Min-max method, localization, quasi newton method

\section{Introduction}

WSNs (Wireless sensor networks) are widely used in various fields because of its convenient data collection and transmission, and simple networking. However, in any field, all kinds of information associated with data are indispensable, and location information is the most important of this information [1]. Therefore, location technology is a hot and difficult point in the research of wireless sensor networks, and it is the basis of acquiring various monitoring objects information [2].

In practice, localization algorithms can be divided into Range-Based algorithm and Range-free algorithm [3]. The latter can only be implemented according to the connectivity, such as convex programming algorithm [4], centroid algorithm [5], APIT 
algorithm [6], DV-Hop algorithm and more [7]. Range-based localization algorithm requires high hardware equipment and multiple measurements to obtain reasonable positioning accuracy, so the computation and communication costs are very high. Compared with the ranging-free algorithm, the location method is simple, does not need additional hardware equipment, and has less communication overhead. Therefore, this location method has attracted more and more attention. Among them, DVHop has many advantages in rang-free algorithm and is widely used in large WSN [8].

DV-Hop is a range-free algorithm that does not require additional hardware to locate only according to network connectivity. It is a coarse-grained estimation and localization algorithm. At present, experts and scholars at home and abroad have tried to improve the DV-Hop algorithm, and a lot of research work has been done. The localization accuracy is much higher than that of traditional algorithm. Liu Yan-hong and Liu Bing-ri of Jilin University proposed to replace the one-hop distance of anchor node in DV-Hop algorithm with RSSI ranging technology and to use hyperbola method instead of trilateral measurement to achieve positioning [9]. To a certain extent, the positioning accuracy has been greatly improved. Su Kai and Cao Yuan of Nanjing University put forward a hybrid location system based on UWB and DGPS [10]. Lin Jin-chao of Chongqing University of Posts and Telecommunications uses Taylor series method to iteratively refine the coordinates of unknown nodes by calculating the initial values of the location results obtained by least square method [11]. The above improvements only improve the DV-Hop algorithm step by step without considering its location accuracy. This paper mainly improves the DV-Hop algorithm from two aspects and simulates the comprehensive improvement. Compared with the original algorithm, the positioning accuracy and stability of the proposed algorithm are greatly improved.

\section{DV-Hop Algorithm Description}

The positioning process is as follows:

Step 1: Distance vector switching protocol: According to the distance vector routing switching protocol, anchor nodes send packets to all other nodes in the network.

Step 2: Calculate the average hop and the distance: After obtaining the coordinates and hops of other anchor nodes, the average hop distance of each anchor node is calculated by formula 1. Through the second flooding method, all the obtained anchor node information and the average hop distance are sent to the network. In order to ensure that the unknown node receives the average hop distance of the nearest anchor node, only the first received average hop distance is saved.

$$
H S_{i}=\frac{\sum_{j \neq i, i=1}^{N}\left(h_{i j} * d_{i j}\right)}{h_{i j}^{2}}
$$


Among them, $d_{i j}=\sqrt{\left(x_{i}-x_{j}\right)^{2}+\left(y_{i}-y_{j}\right)^{2}}$ and $\left(x_{i}, y_{i}\right),\left(x_{j}, y_{j}\right)$ is the coordinate between $i, j . h_{i j}$ is the minimum hop $(i \neq j)$. When the average hop distance of anchor node is obtained from formula 1, the estimated distance can be obtained by average hop distance and hop count [12].

Step 3 Positioning stage: The estimated distance between anchor node and unknown node is obtained by the second stage, so it can be solved by node coordinate estimation algorithm.

\section{Improved Algorithm-CNDV-Hop (Corrected Quasi Newton DV-Hop)}

In this paper, CNDV-Hop algorithm is simulated from the following two aspects and the positioning accuracy are greatly improved.

\subsection{Improved average hopping distances}

The traditional algorithm makes the unbiased estimation criterion calculate the average distance per hop. However, in random distributed wireless sensor networks, the estimation error of average hop distance is Gaussian distribution, and it is reasonable to use mean square error to estimate the average hop distance [13]. Therefore, the minimum mean square error criterion is used to calculate the distance instead of the unbiased estimation criterion.

Namely,

$$
f_{\varepsilon}=\sum_{j \neq i}\left(d_{i j}-H S_{i} \cdot h_{i j}\right)^{2}
$$

Let $\frac{\partial f_{\varepsilon}}{\partial H S_{i}}=0$, the average hop distance of anchor node $i$ can be re-represented as:

$$
H S_{i}=\frac{\sum_{j \neq i, i=1}^{N}\left(h_{i j} * d_{i j}\right)}{h_{i j}^{2}}
$$

The average hop distance can be recalculated by formula 1, the minimum hop number between $i$ and $j$ are three hops; between $j, k$ are three hops, and between $i, k$ are six hops. The average hop distance $i, j, k$ are: 


$$
\begin{aligned}
& H S_{i}=\left(3 d_{i j}+6 d_{i k}\right) /\left(3^{2}+6^{2}\right) \\
& H S_{j}=\left(3 d_{j i}+3 d_{j k}\right) /\left(3^{2}+3^{2}\right) \\
& H S_{k}=\left(6 d_{k i}+3 d_{k j}\right) /\left(6^{2}+3^{2}\right)
\end{aligned}
$$

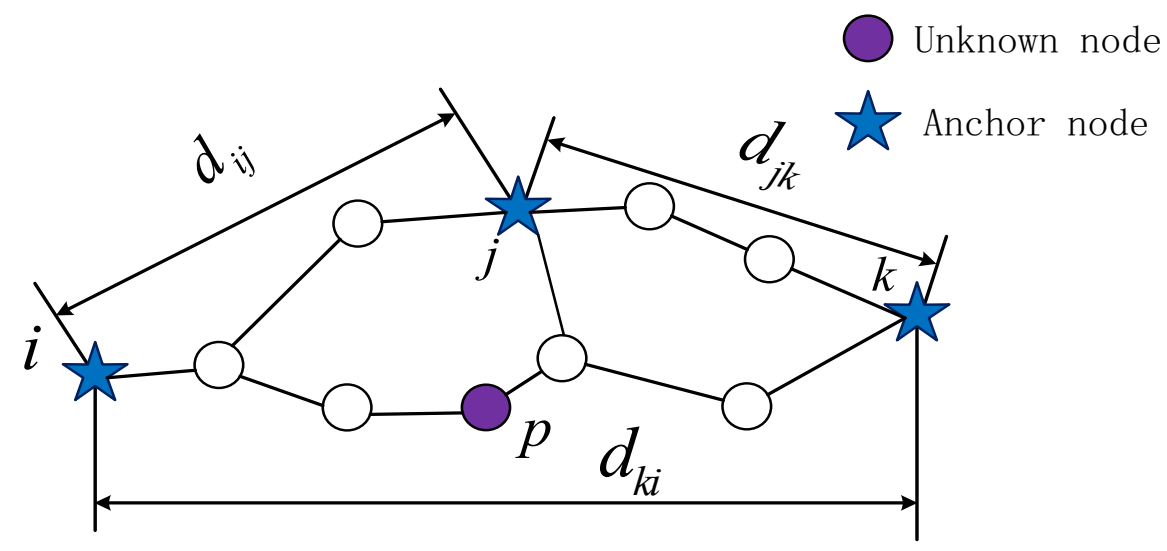

Fig. 1. Network topology diagram

In the second phase, the unknown node regards the first anchor node's average hop distance as its own. Considering the hop distance information of multiple anchor nodes, the positioning accuracy can be improved [14]. The number of anchor nodes that can communicate with unknown nodes is $\mathrm{k}$, and the average hop correction for $\mathrm{k}$ anchor nodes is:

$$
\varpi=\frac{\sum_{i=1}^{i=k} H S_{i}}{k}
$$

Combined with formula (4), the improved average hop distance is as follows:

$$
H S_{i}^{\prime}=\frac{H S_{i}+\varpi}{2}
$$

In the formula, the improved average hop distance will be closer to the actual average hop distance of the whole network if the anchor node participates in the modification and improvement of calculating the average hop distance.

From the above conclusions and combined with Figure 1, the average hop distance of the improved anchor nodes $i, j, k$ are: 


$$
H S_{i}^{\prime}=\left(H S_{i}+\varpi\right) / 2, H S_{j}^{\prime}=\left(H S_{j}+\varpi\right) / 2, H S_{k}^{\prime}=\left(H S_{k}+\varpi\right) / 2 \text { Therefore, }
$$

$p$ to the anchor nodes $i, j, k$ are estimated to be: $d_{p i}=3 \times H S_{i}^{\prime}, d_{p j}=2 \times H S_{j}^{\prime}$ and $d_{p k}=3 \times H S_{k}^{\prime}$.

\subsection{Improvement of positioning calculation}

Performance comparison between ML algorithm and Min-Max algorithm: Traditional position calculation methods mostly use trilateral location method or ML algorithm to obtain coordinates [12]. Trilateral location method is simple in calculation and low in positioning accuracy. Maximum likelihood estimation method relies on ranging error, which requires a large number of floating-point operations and a large amount of computation. The biggest advantage of Min-Max algorithm is that it can obtain better positioning results without a lot of calculation, but the accuracy is not high when the number of anchor nodes is large. Based on this conclusion, the paper proposes that when the number of anchor nodes is small, the minimummaximum method is used to obtain the estimated coordinates, and when the number of anchor nodes is high, the maximum likelihood method is used to obtain the coordinates. Finally, the quasi Newton method is used to iteratively optimize the estimated coordinates.

In order to verify the positioning accuracy of anchor node by using maximum size (Min-Max) and maximum likelihood estimation (ML), this two kinds of positioning algorithms are simulated and analyzed in this paper. The simulation area is set to 200 * 200, simulation 100 times to take the mean.

Assuming that the communication radius is $40 \mathrm{~m}, 20$ random nodes are randomly deployed in the $200 * 200$ meter area. In Figure 2 and Figure 3, 15 and 6 anchor nodes are respectively deployed. The simulation uses Min-Max method and ML method to solve unknown nodes. It can be seen from these two figures that ML positioning method has higher accuracy when there are more anchor nodes, and the highest accuracy is about 20 meters higher than Min-Max method. Min-Max positioning accuracy is higher than ML method when there are fewer anchor nodes, and the highest accuracy is about 50 meters. It can be seen that in the case of low proportion of anchor nodes, low computational complexity and low computational load, Min-Max method is usually used, which not only reduces the communication cost, but also reduces energy consumption. When the proportion of anchor nodes is high, ML method should be used to improve the positioning accuracy. Therefore, when solving unknown node coordinates, it is necessary to select the appropriate algorithm according to the network deployment. 


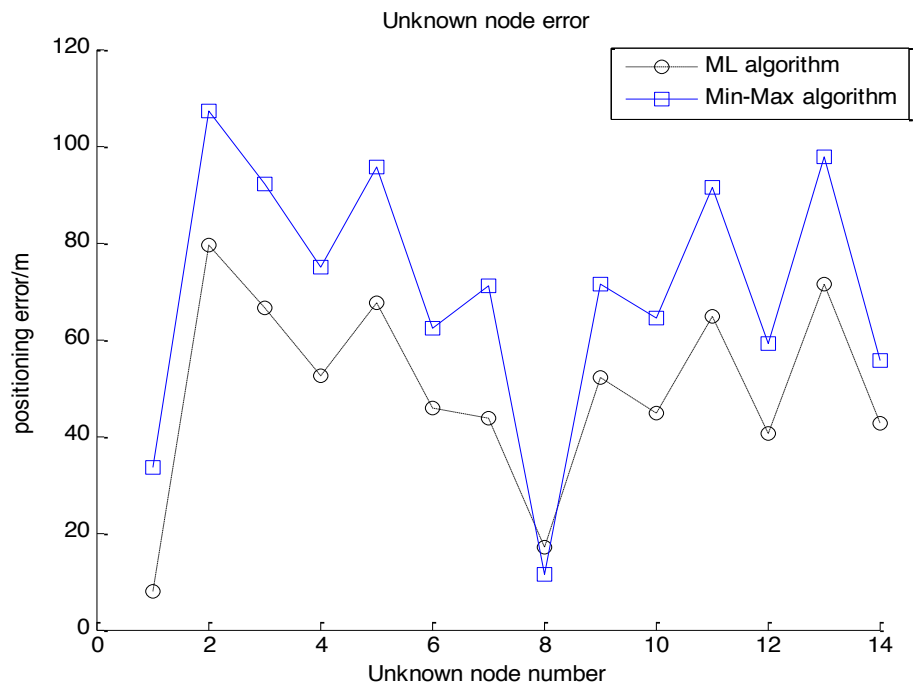

Fig. 2. Error comparison of two localization algorithms when the anchor node is 15

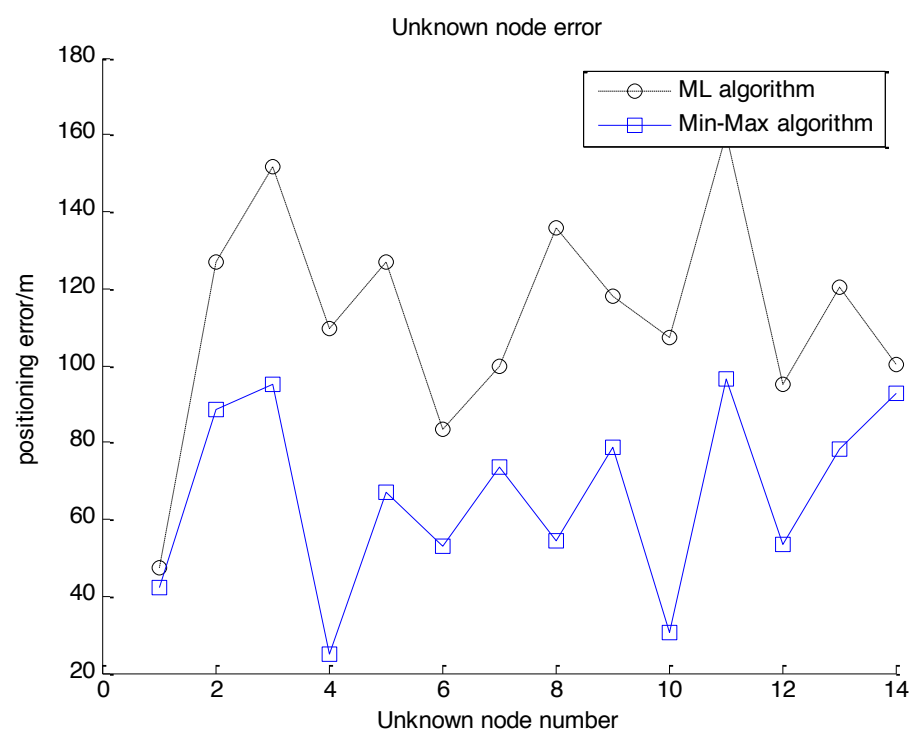

Fig. 3. Error comparison of two localization algorithms when anchor nodes are 6

In order to further determine the relationship between the ratio of anchor node and the accuracy of the two algorithms, the normalized average positioning error of the two algorithms is simulated in Figure 4 when the number of anchor nodes is increased from 3 to 11. It can be seen from the figure that ML algorithm more dependent on the 
ratio of anchor nodes. When the number of anchor nodes is small, the error of ML algorithm is large.

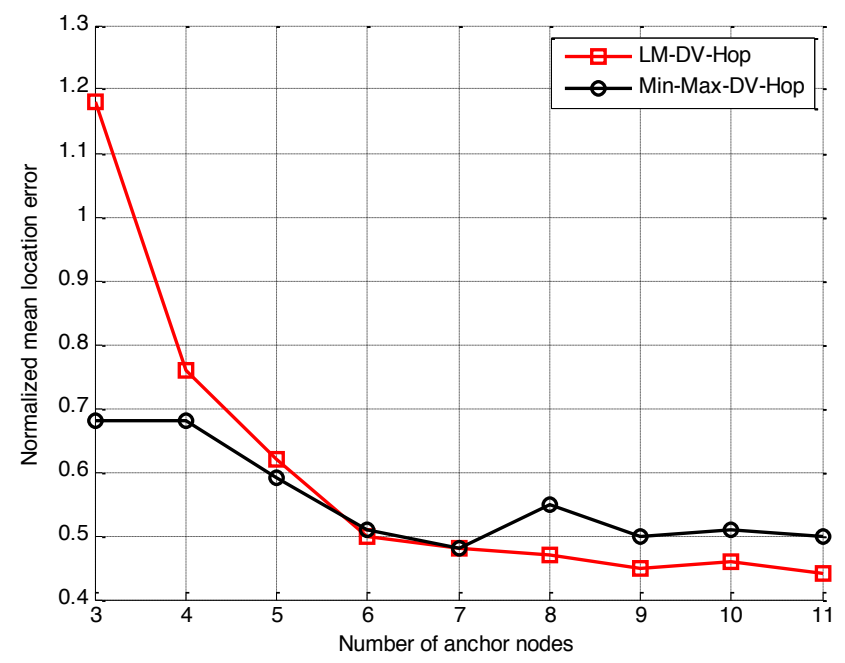

Fig. 4. Contrast Diagram of Two Algorithms with the Number of Anchor Nodes

Table 1. Comparison of the two algorithms of normalized average localization error

\begin{tabular}{|c|c|c|c|c|}
\hline \multirow{2}{*}{ Communication radius } & \multicolumn{2}{|c|}{ Proportion of Anchor nodes 20\% } & \multicolumn{2}{c|}{ Proportion of Anchor nodes 30\% } \\
\cline { 2 - 5 } & $\begin{array}{c}\boldsymbol{M L} \boldsymbol{D} \boldsymbol{D} \boldsymbol{V}- \\
\boldsymbol{H o p}\end{array}$ & Min-Max-DV-Hop & $\boldsymbol{M L}$-DV-Hop & $\begin{array}{c}\text { Min-Max-DV- } \\
\boldsymbol{H o p}\end{array}$ \\
\hline 35 & 1.0520 & 0.9320 & 0.8576 & 0.8697 \\
\hline 40 & 0.8176 & 0.8152 & 0.6641 & 0.6527 \\
\hline 45 & 0.7158 & 0.6315 & 0.5469 & 0.5221 \\
\hline 50 & 0.6310 & 0.6023 & 0.4432 & 0.4815 \\
\hline 55 & 0.5178 & 0.5680 & 0.3928 & 0.4361 \\
\hline
\end{tabular}

As shown in Table 1, the better the performance of Min-Max-DV-Hop algorithm is, the lower the proportion of anchor nodes is, the higher proportion of anchor nodes is, and the better performance of ML-DV-Hop algorithm is. The network area is $200 * 200 \mathrm{~m}$, and the critical proportion of anchor nodes is $30 \%$ when the total number of nodes is 20 .

Iterative refinement based on Quasi Newton method: The distance between the anchor node and the unknown node is found. Assuming that the unknown node in $\mathrm{K}$ anchor nodes participates in the localization, the equation set shown in (6) can be used.

When the distance between the anchor node and the unknown node is found, if there are $\mathrm{k}$ anchor nodes in which the unknown node $\mathrm{p}$ participates in, the equation (6) can be used. 


$$
\left\{\begin{array}{c}
\left(x_{1}-x\right)^{2}+\left(y_{1}-y\right)^{2}=d_{1}^{2} \\
\vdots \\
\left(x_{k}-x\right)^{2}+\left(y_{k}-y\right)^{2}=d_{k}^{2}
\end{array}\right.
$$

The equation (6) is transformed into a nonlinear optimization problem, namely:

$$
\left.\begin{array}{l}
f(x, y)=\sum_{i=1}^{k}\left(\left(x_{i}-x\right)^{2}+\left(y_{i}-y\right)^{2}-d_{i}^{2}\right)^{2} \\
\min f(x, y)
\end{array}\right\}
$$

Among them, the coordinates of anchor node $i$ are $\left(x_{i}, y_{i}\right) ; d_{i}$ is the distance between unknown node and anchor node. For the optimization problem, it needs to obtain the optimal solution $X^{*}\left(x^{*}, y^{*}\right)$ of the objective function $f(x, y), x \in R^{+}, \mathrm{y} \in R^{+}$.

In this algorithm, in order to make the positioning accuracy higher and solve the objective function quickly, the estimated coordinate $X^{(0)}\left(x_{0}, y_{0}\right)$ is set as the initial point approximation of the Quasi-Newton algorithm firstly, and $\left\|f\left(x^{k+1}\right)\right\| \leq \varepsilon$ is regarded as the convergence criterion of the algorithm. A new approximation point $X^{(1)}\left(x_{1}, y_{1}\right)$ is obtained and the gradient fall of $f(x, y)$ is guaranteed until the optimal solution $X^{*}\left(x^{*}, y^{*}\right)$ is obtained by finite iteration [12].

\section{$3 \quad$ Simulation and Experiment}

\subsection{Simulation environment and evaluation indicators}

In this paper, the improved positioning algorithm is simulated by using MATLAB simulation platform, and compared with the classical improved DV-Hop algorithm in recent two years. Reference [15] is recorded as ORDV-Hop. The performance of the algorithm can be fully demonstrated by simulating and analyzing the simulation environment, topology and node characteristics of wireless sensor networks. The network topology is set as follows: 100 nodes are randomly generated in the network, and the network parameters such as the sum-up points, anchor nodes and communication radius are the same in each simulation.

CNDV-Hop algorithm is evaluated by calculating the average location error, the normalized average location error, the root mean square error by equations

$$
A E=\frac{\sum_{j=1}^{N} \sum_{i=1}^{K}\left|T_{i}-\tilde{T}_{i}\right|}{K N}, \quad N A E=\frac{A E}{R} \text { and } N R M S E=\sqrt{\frac{\sum_{i=M}^{N}(e(i)-r(i))^{2}}{N \times R^{2}}}
$$




\subsection{Simulation analysis}

Figure 5 is the topological structure of wireless sensor networks, which is set as $200 * 200$, and the area is distributed with 100 nodes randomly. Figures 6 and Figure 7 simulate the deviation between the actual and estimated positions of the unknown nodes in DV-Hop and CNDV-Hop algorithms respectively. From these two figures, it can be seen that the localization accuracy of CNDV-Hop algorithm is significantly higher than DV-Hop algorithm.

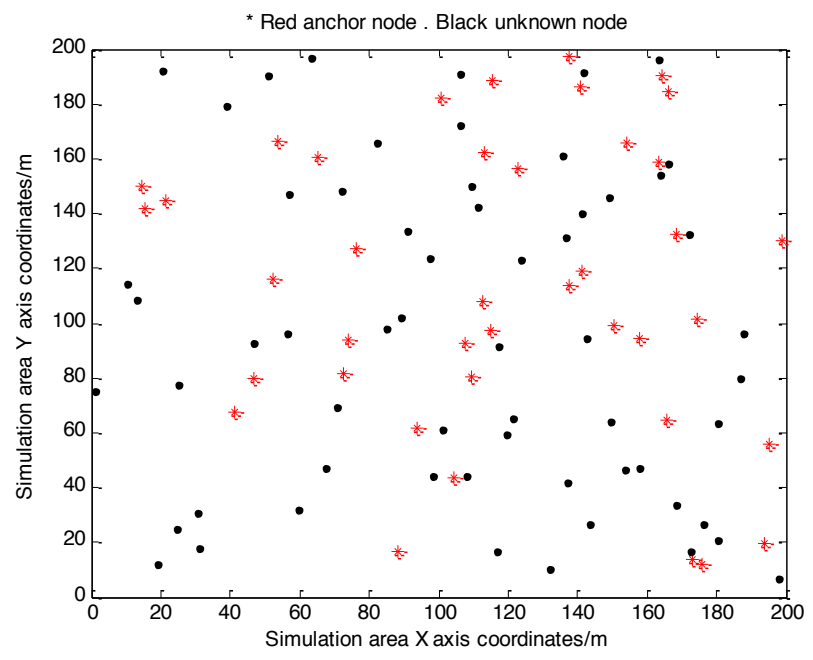

Fig. 5. Random distributions of network nodes

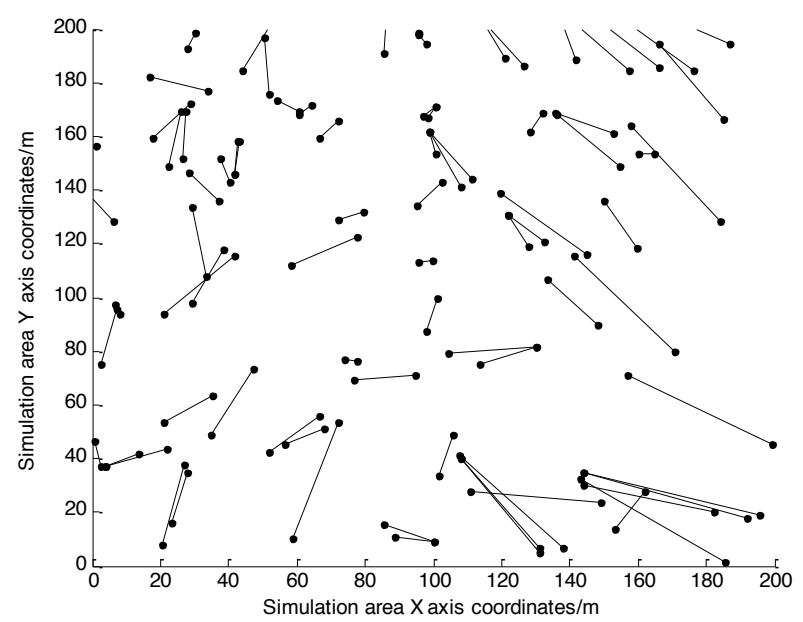

Fig. 6. Error chart for the Actual Position and Estimated Position of DV-Hop Algorithms 


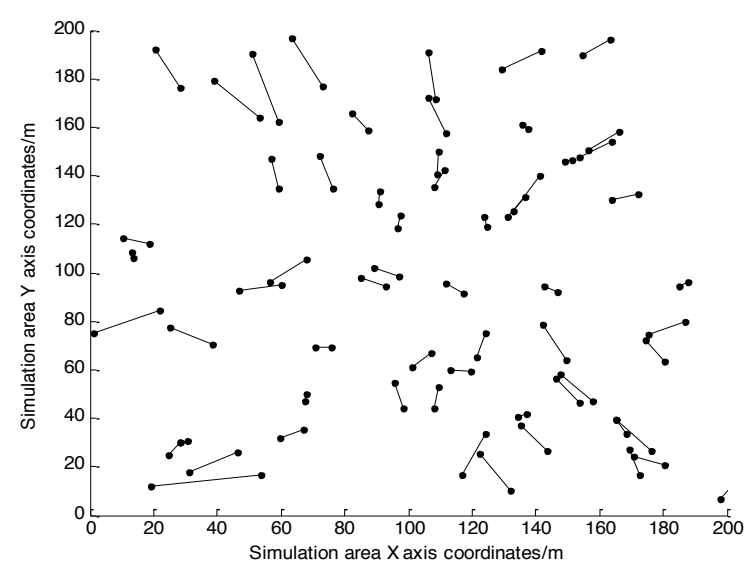

Fig. 7. Error chart for the Actual Position and Estimated Position of CNDV-Hop Algorithms

In Figure 8, it sets the ratio of anchor nodes is $30 \%$. When $\mathrm{R}$ (communication radius) is 30 meters, the influence of the anchor node on the positioning results is simulated in different proportions. It can be calculated from the simulation that the normalization error of DV-Hop algorithm is 0.5164; the ORDV-Hop algorithm is about 0.4147 and the CNDV-Hop algorithm is 0.3829. In Figure 9, when R is 40 meters. The normalization error of DV-Hop algorithm is about 0.2958 , and the ORV-Hop algorithm is about 0.2530 . However, CNDV-Hop algorithm proposed in the paper is only 0.1753 . In Figure 10, the proportion of anchor nodes is $30 \%$. The simulation results show that the normalized root mean square error of CNDV-Hop algorithm is the lowest. When the communication radius is 40 , the normalized root mean square error of DV-Hop algorithm is 0.3221 , while the normalized root mean square error of CNDV-Hop algorithm is only 0.1765 .

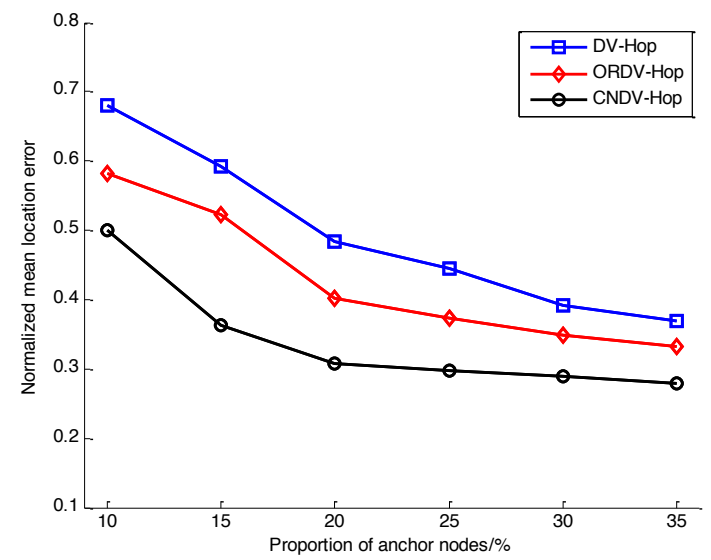

Fig. 8. The influence of anchor node proportion change on positioning error when $\mathrm{R}$ is 30 


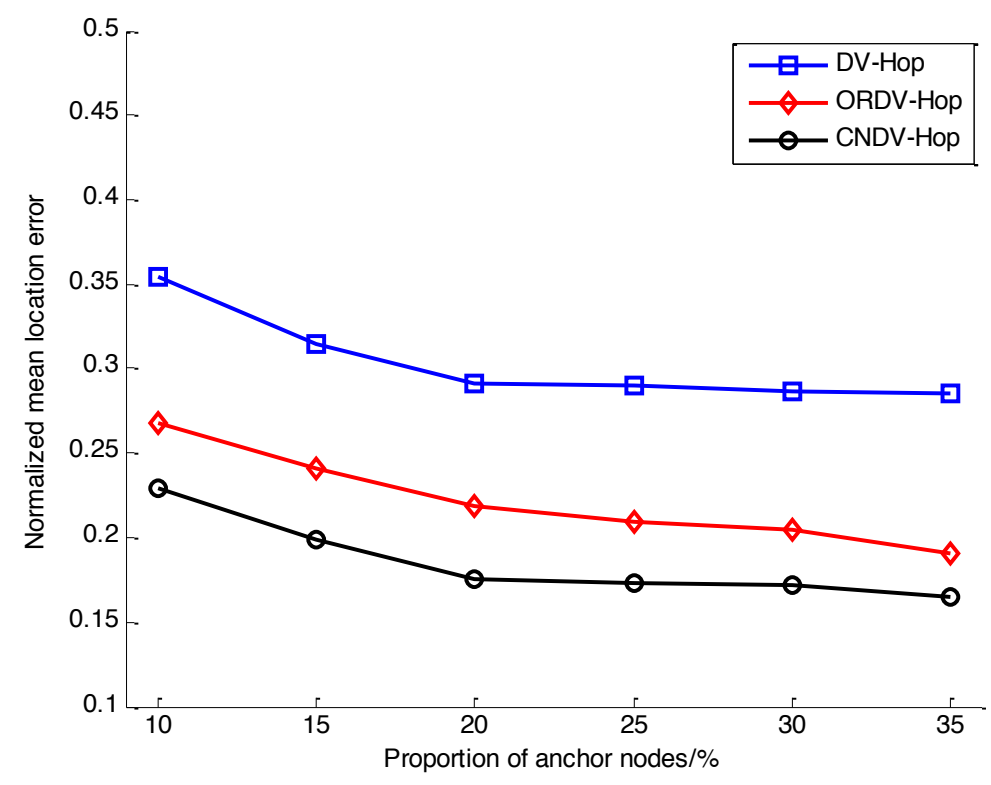

Fig. 9. The influence of anchor node proportion change on positioning error when $\mathrm{R}$ is 40

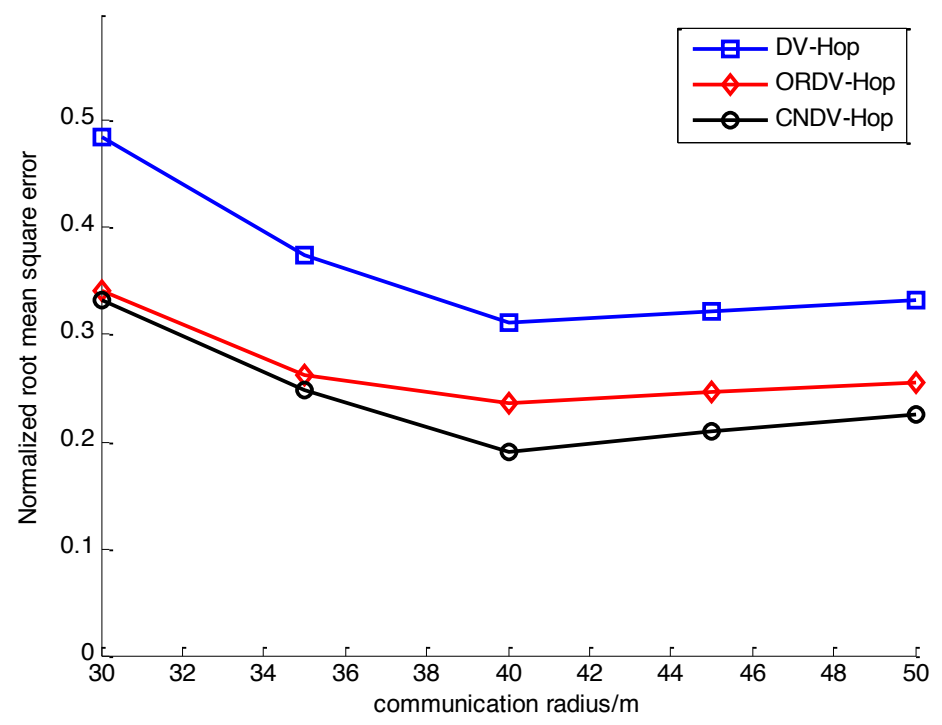

Figure 10 The influence of communication radius on root mean square error when the proportion of anchor nodes is $40 \%$. 


\section{Conclusion}

In this paper, a WSN localization algorithm based on DV-Hop is proposed, and the average hop distance and location calculation are corrected respectively. The improved average hop distance makes full use of the optimized anchor node information and has higher positioning accuracy than single anchor node. In the location calculation method, the improvements of the algorithm mainly include initial value estimation and final estimation of node coordinates. In the initial estimation, when there are fewer anchor nodes, Min-Max algorithm is used to solve the problem, and ML algorithm is used to solve the problem when there are more anchor nodes. At this time, through simulation, the localization algorithm of the proportion of anchor nodes in different situations is given. In the final estimation, the Quasi-Newton algorithm is used to iteratively optimize the initial value. Experiments show that the algorithm is a simple and efficient ranging-free positioning algorithm for wireless sensor networks.

\section{Acknowledgement}

The work was supported by Projects of Natural Science Foundational in Higher Education Institutions of Anhui Province (KJ2017A449);

\section{References}

[1] Zhong-min Pei, Yi-bin Li, Shuo Xu. (2013). A fast localization algorithm for large-scale wireless sensor networks, Journal of China University of Mining \& Technology, Vol. 42(2), pp. 314-319

[2] En-jie Ding, Xin Qiao, Fei Chang. (2014). Improved Weighted Centroid Localization Algorithm Based on RSSI Differential Correction, International Journal on Smart Sensing and Intelligent Systems, Vol. 7(3), pp. 1156-1173 https://doi.org/10.21307/ijssis-2017-699

[3] Capkun S, Hamdi M, Hubaux J P. (2001). GPS-free positioning in mobile Ad-Hoc networks, Proc Hawaii International Conference on System Sciences, Maui, HW, USA, pp. 3481-3490.

[4] Doherty L, Pister K, and Ghaoui L, (2001). Convex Position Estimation in Wireless Sensor Networks, Proc. of the IEEE INFOCOM, Anchorage, AK, USA, pp. 1655-1663. https://doi.org/10.1109/INFCOM.2001.916662

[5] Harter A, Hopper A, Steggles P, (2002). The Anatomy of a Context-Aware Application, Wireless Network, pp. 187-197. https://doi.org/10.1023/A:1013767926256

[6] He T, Huang C D, Blum B M, (2003). Range-Free Localization Schemes for Large Scale Sensor Networks, Proceedings of the Ninth Annual International Conference on Mobile Computing and Networking San Diego, United states, pp. 81-95. https://doi.org/10. $1145 / 938985.938995$

[7] Niculescu D,Nath B, (2003). DV Based Positioning in Ad Hoc Networks, Journal of Telecommunication Systems, Vol. 22(1-4), pp. 267-280. https://doi.org/10.1 $\underline{\text { 023/A: } 1023403323460}$ 
[8] Xin Qiao, Han-Sheng Yang, Zheng-Chuang Wang, (2017). Iterative L-M Algorithm in WSN - Utilizing Modifying Average Hopping Distances, International Journal of Online Engineering, Vol. 10(13), pp. 4-20. https://doi.org/10.3991/ijoe.v13i10.7006

[9] Yan-heng Liu, Bing-ri Liu, etc, (2010). Improved DV-Hop algorithm in localization accuracy in WSN, Journal of Jilin University (Engineering and Technology Edition), Vol. 40(3), pp. 763-768.

[10] Kai Su, Yuan Cao, etc, (2010). On hybrid localization based on UWB and DGPS, Computer Applications and Software, Vol. 27(5), pp. 212-215.

[11] Jin-Zhao Lin, Xiao-Bing Chen, Hai-Bo Liu, (2009). Iterative algorithm for locating nodes in WSN based on modifying average hopping distances, Journal on Communications, Vol. 30(10), pp. 107-113.

[12] Xin Qiao, Fei Chang, En-Jie Ding, (2014). Modifying Average Hopping Distances Based Iterative Algorithm for Quasi-Newton in WSN, Chinese Journal of Sensors and Actuators, Vol. 27(6), pp. 797-801.

[13] Wei-Wei JI, Zhong Liu. (2008). Study on the application of DV-Hop localization algorithms to random sensor networks, Journal of Electronics\& Information Technology, 30(4):970-974.

[14] Xin Qiao, (2015). Localization Algorithm of Wireless Sensor Network and Its Improvement Based on DV-Hop, China University of Mining and Technology, Xuzhou.

[15] Yu-Cheng Wu, Jiang-Wen Li, (2012). Improved DV-Hop Localization Algorithm Based on Optimal Communication Radius of Nodes, Journal of South China University of Technology (Natural Science Edition), Vol. 40(6), pp. 36-42.

\section{$7 \quad$ Authors}

Ms. Xin-Qiao, was born in 1988, Suzhou City, Anhui Province, China. She is a Lecturer and having a Master's degree. She has been published a number of highlevel papers and her research interests are wireless sensor network positioning technology, ZigBee technology etc. She is with School of Electronic Engineering, Chaohu University, Chaohu, 238000, Anhui, China.

Fei-Chang is with Inspur Group Company Limited, Jinan, 250000, Shandong, China.

Jing-Ling is with School of Electronic Engineering, Chaohu University, Chaohu, 238000, Anhui, China.

Article submitted 2018-10-10. Resubmitted 2019-01-06. Final acceptance 2019-01-11. Final version published as submitted by the authors. 